\title{
Application of the theory of damping of kink oscillations by radiative cooling of coronal loop plasma
}

\author{
R. J. Morton and R. Erdélyi
}

Solar Physics and Space Plasma Research Centre ( $\left.\mathrm{SP}^{2} \mathrm{RC}\right)$, University of Sheffield, Hicks Building, Hounsfield Road, Sheffield S3 7RH, UK

e-mail: [app07rjm;Robertus]@sheffield.ac.uk

Received 25 March 2010 / Accepted 18 May 2010

\begin{abstract}
Aims. We present here a first comparative study between the observed damping of numerous fast kink oscillations and the theoretical model of their damping due to the cooling of coronal loops. The theory of damping of kink oscillations due to radiation of the solar plasma with a temporally varying background is applied here to all known cases of coronal kink oscillations.

Methods. A recent dynamic model of cooling coronal loops predicts that transverse oscillations of such loops could be significantly damped due to the radiative cooling process (Morton \& Erdélyi 2009, ApJ, 707, 750). The cooling of the loop plasma also has the consequence that the kink oscillation has a time-dependent frequency. The theory is applied to a relatively large number of known and reported examples of TRACE observations of damped kink oscillations.

Results. We find that, for cooling timescales that are typical of EUV loops (500-2000 s), the observed damping of the transversal (i.e. kink) oscillations can be accounted for almost entirely by the cooling process in half of the examples. No other dissipative mechanism(s) seems to be needed to model the damping. In the remaining other examples, the cooling process does not appear to be able to account fully for the observed damping, though could still have a significant influence on the damping. In these cases another mechanism(s), e.g. resonant absorption, may be additionally required to account for the complete decay of oscillations. Also, we show that because of the dynamic nature of the background plasma, allowing for a time-dependent frequency provides a better fit profile for the data points of observations than a fit profile with a constant frequency, opening novel avenues for solar magneto-seismology.
\end{abstract}

Key words. magnetohydrodynamics (MHD) - plasmas - Sun: corona - waves

\section{Introduction}

The first spatially resolved oscillations in coronal loops where reported with the Transitional Region And Coronal Explorer (TRACE) satellite (e.g. Aschwanden et al. 1999). Due to the transverse nature of these oscillations, they where identified as the fast kink body magnetohydrodynamic (MHD) mode (Nakariakov et al. 1999). Since then, there have been a relatively large number of observations of kink oscillations making them a useful tool for diagnostics of the coronal plasma (see e.g. Andries et al. 2009; and Ruderman \& Erdélyi 2009, for the most recent reviews on kink waves). One outstanding problem associated with the kink oscillations is that they are observed to be heavily damped, usually within $4-5$ periods. The cause of the damping is, to date, still unknown. Resonant absorption is thought to be a strong candidate (Ruderman \& Roberts 2002; Goossens et al. 2002) and in certain cases may provide an explanation for the observed damping (Arregui et al. 2007; Goossens et al. 2008). A working alternative is shown here.

In general, models of coronal loops that support MHD oscillations have been assumed to be static with respect to the background plasma quantities. However, the solar corona is known to be of a highly dynamic nature. One such dynamic feature which is ubiquitous and dominant in the corona and has been observed on numerous occasions is the cooling of coronal loops (e.g. López Fuentes et al. 2007; Aschwanden \& Terradas 2008).

It was preferentially thought, up until fairly recently, that coronal loops existed in a state close to that of static equilibrium, where the heating balanced the cooling of the plasma.
However, the static equilibrium models could not reproduce the observed properties of large EUV coronal loops (Aschwanden et al. 2000a). On the other hand, thermal non-equilibrium models are found to be able to account for many observed properties (see, e.g. Klimchuk et al. 2009). These models suggest that coronal loops undergo cycles of heating and cooling phases over their lifetimes, with the heating phase being rapid compared to the slow cooling phase. This process has been observed on numerous occasions. One such typical example is coronal loops being heated to soft $\mathrm{X}$-ray temperatures, $T>2 \mathrm{MK}$, before cooling down through to the EUV temperature range, $T \approx 1-2 \mathrm{MK}$ (Nagata et al. 2003; Winebarger \& Warren 2005; Taroyan et al. 2007). Further, Reale et al. (2000) reported the complete heating and cooling of a loop within the EUV temperature range.

Loops can also experience a localised heating, e.g. due to flares, which are also the suggested drivers for EUV kink oscillations. Simulations by Jakimiec et al. (1992) of flaring loops show the loops experience an initial, short lived heating phase followed by a slow cooling phase. There is mounting observational evidence for this phenomena. Ofman \& Wang (2008) and Erdélyi \& Taroyan (2008) both observed separate flaring events taking place in the vicinity of loop footpoints. The heating and cooling phases where identified in both observations and transverse oscillations in the loops where also reported.

Cooling timescales for EUV loops have been estimated in Aschwanden \& Terradas (2008) to be around 500-2000 s. These cooling timescales are mostly larger though sometimes comparable to the characteristic timescale of the transverse oscillations, 
e.g. for fast kink body modes the oscillations have typical periods of 300-500 s and last for four to five periods, so the cooling of the loops is expected to influence the oscillations and the use of static background in the modelling of coronal loops is not necessarily justified (Aschwanden \& Terradas 2008).

A new theoretical model of oscillating cooling coronal loops is developed by Morton \& Erdélyi (2009, ME09 hereafter) who suggest that as a coronal loop cools, transverse MHD oscillations of the loops are considerably damped by the cooling of the plasma (see, ME09 for full details on the theoretical model and assumptions made). Here we put the theory outlined by ME09 to a rigorous test by applying it to 27 known examples of damped kink oscillations observed with TRACE and compare the observed rate of damping with the theoretically predicted damping due the cooling of the loop. Next, we also progress by proposing to investigate the time-dependent nature of oscillatory frequencies, opening new avenues in solar magneto-seismology. Often in reports of coronal loop oscillations it is very hard to distinguish between various modes of oscillations (i.e. fundamental, first harmonic, etc.) being present in the system as the mode periods evolve during the observations. Such time dependent periods are found in wavelet analysis (e.g. De Moortel et al. 2004). Strong dynamical behavior in the system could lead to the misinterpretation of data as showing many harmonics when in fact it is just one mode experiencing a change in period.

\section{Key points of theory}

Here we only recall very briefly the key points and results of the theory describing kink oscillations in a cooling coronal loop developed by ME09 that are essential to test the theory. The loop is considered to be longitudinally stratified with a semi-circular geometry in a gravitationally stratified atmosphere. The temperature of the loop is assumed to be isothermal and to evolve over time with an exponential profile. These assumptions serve as a good working approximation of the observed cooling of loops (Aschwanden \& Terradas 2008; Ugarte-Urra et al. 2009), where the important new physics is still captured. A microphysical process of cooling (i.e. thermal conduction or radiation) is ignored and the effect of cooling on the density profile of the loop is concentrated on. The solution to the equation of motion for MHD plasmas with a time dependent density profile requires that the loop has a background flow. This dynamic background profile of the loop causes the loop to be slowly evacuated as it cools and the plasma flows out of the loop at the footpoints. Note, this is a strongly non-conservative physical system.

The radial velocity component in transversal oscillations in cylindrical geometry takes the form,

$v_{\mathrm{r}}=\mathfrak{R}\left\{Q(z, t) \exp \left(\mathrm{i} \int \omega \mathrm{d} t\right)\right\}$.

Here $Q(z, t)$ is the dynamic amplitude, $\omega$ is the frequency of the oscillations and $\mathfrak{R}$ refers to the real part of $v_{r}$. The leading order approximation of the governing equation obtained is

$\frac{\partial^{2} Q(z, t)}{\partial z^{2}}+\frac{\omega^{2}}{c_{k}^{2}(z, t)} Q(z, t)=0, \quad Q(z, t)=0$ at $z= \pm L$,

where $c_{\mathrm{k}}^{2}(z, t)$ is the time-dependent kink speed. Equation (2) is a generalised time-dependent Sturm-Liouville equation, for its static counterpart see, e.g. Dymova \& Ruderman (2005), Verth \& Erdélyi (2008). The validity of the approximate solution to Eq. (2), is subject to the condition that the cooling timescale, $\tau_{\text {cool }}$, is greater than the characteristic period, $P$, of the oscillation, i.e. $P / \tau_{\text {cool }}<1$. It should be noted here that the smaller the value of $P / \tau_{\text {cool }}$ the greater the accuracy of the solution. However, a value of $P / \tau_{\text {cool }}$ of the order one can still provide an adequate approximation (see, e.g. Bender \& Orszag 1978).

There are a number of interesting effects on the transverse oscillations as the loop cools. Solutions to Eq. (2) show that as the loop cools, transverse oscillations of the loop are damped due to the cooling process. The rate of damping was also found to dependent upon the initial temperature of the loop as the oscillation started, the height of the loop apex in the atmosphere and the cooling timescale, $\tau_{\text {cool }}$. Another point that should be emphasised and is important to applications of solar magnetoseismology is the change in period of the modes. An analytic expression can be found for the period change if a solution to Eq. (2) is sought using the variational approach suggested by, e.g. McEwan et al. (2006). Assuming that $h_{\mathrm{a}} / H \ll 1$ where $h_{\mathrm{a}}$ is height of loop apex above photosphere and $H$ is the scale height, then it is obtained for the fundamental mode

$$
P_{1} \approx \frac{1}{c_{\mathrm{kf}}} \frac{4 L}{\pi^{3 / 2}}\left[\frac{\pi}{4}-\frac{2 h_{\mathrm{a}}}{141 T(0)}\left[\frac{\mathrm{MK}}{\mathrm{Mm}}\right]\left(1+\delta \frac{t}{P}\right)\right]^{\frac{1}{2}},
$$

where $c_{\mathrm{kf}}$ is the kink speed at the loop foot points. It can clearly be seen that the period decreases as time increases, i.e. the loop cools. Let us check the observational signatures of these analytical predictions.

\section{Application to observed kink oscillations}

Here we apply the theory to the 27 known damped kink oscillations available in the current literature. In Fig. 1, we show selected typical observations that have been identified as damped fast kink oscillations, each of them observed by the TRACE satellite. A catalogue of these observations is available in Nakariakov et al. (1999) and Aschwanden et al. (2002). In order to apply the theory, relevant information on the oscillations and their damping characteristics is given in Table 1. The data points from the observations have been fitted initially with a damped sine function of the form

$f(t)=A_{0} \sin (\omega t-\theta) \exp \left(-t / t_{\mathrm{d}}\right)$,

where $\theta$ is the phase shift, $A_{0}$ is the amplitude at $t=0$ and $\tau_{\mathrm{d}}$ is the damping time. This fitting process allows us to determine the damping time and its approximate relation to the period of oscillations. Estimated values of the period $P$, damping time, phase shift and initial amplitude for each observation are given under the corresponding fitting of each oscillation in Fig. 1.

The damped oscillating coronal loops all appeared in the TRACE EUV filters, either in the $171 \AA$ or the $195 \AA$ filter. The filters have a broad temperature response where the $171 \AA$ filter has a peak response at $T \approx 0.96 \mathrm{MK}$ and the $195 \AA$ at $T \approx 1.37 \mathrm{MK}$ (Aschwanden et al. 2000b). This suggests that the loop enters the $195 \AA$ filter at a temperature of around $1.5 \mathrm{MK}$, so we set the initial temperature of the loop to about $1.5 \mathrm{MK}$ as the oscillation begins and model a number of cooling timescales that are representative of EUV loops, i.e. 500-2000 s. The height of the loop apex is also required to determine the fundamental period (see Eq. (3)) and is given in Table 1. The damping profile is obtained by calculating the value of $v_{\mathrm{r}}$ at the loop apex, i.e. the maximum value of velocity for the fundamental mode, and normalising with respect to the initial value of $v_{\mathrm{r}}(0)$, i.e. the value at $t=0$. The normalised damping profile is then scaled by the 


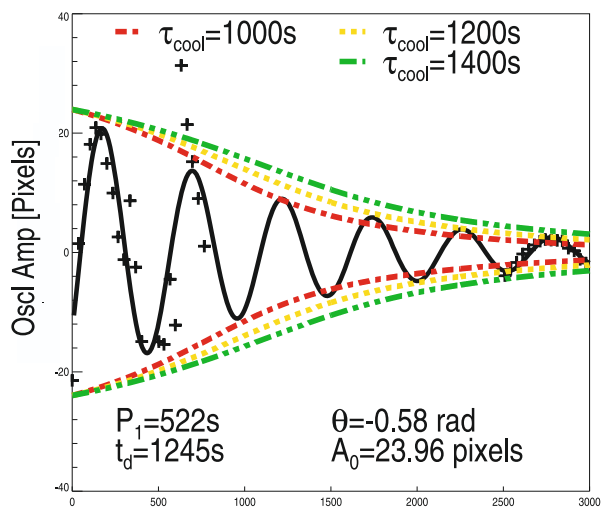

$t(s)$

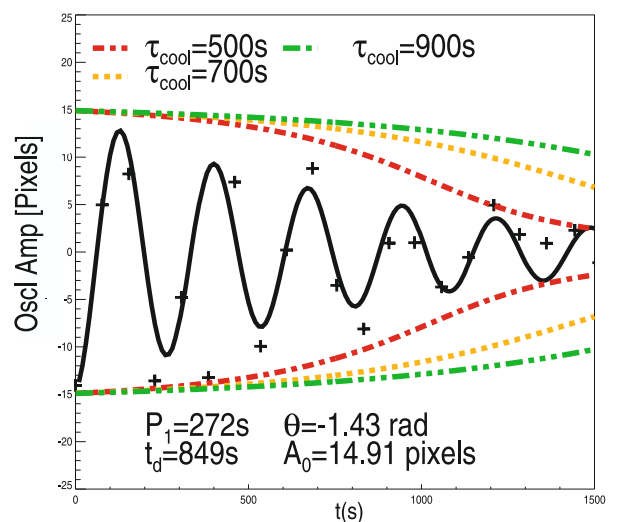

$t(s)$
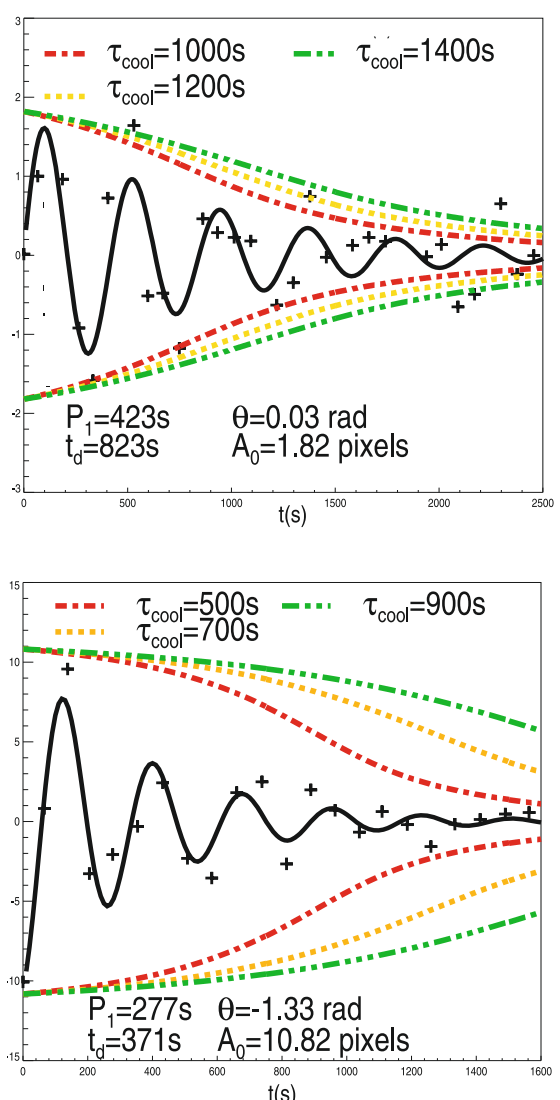
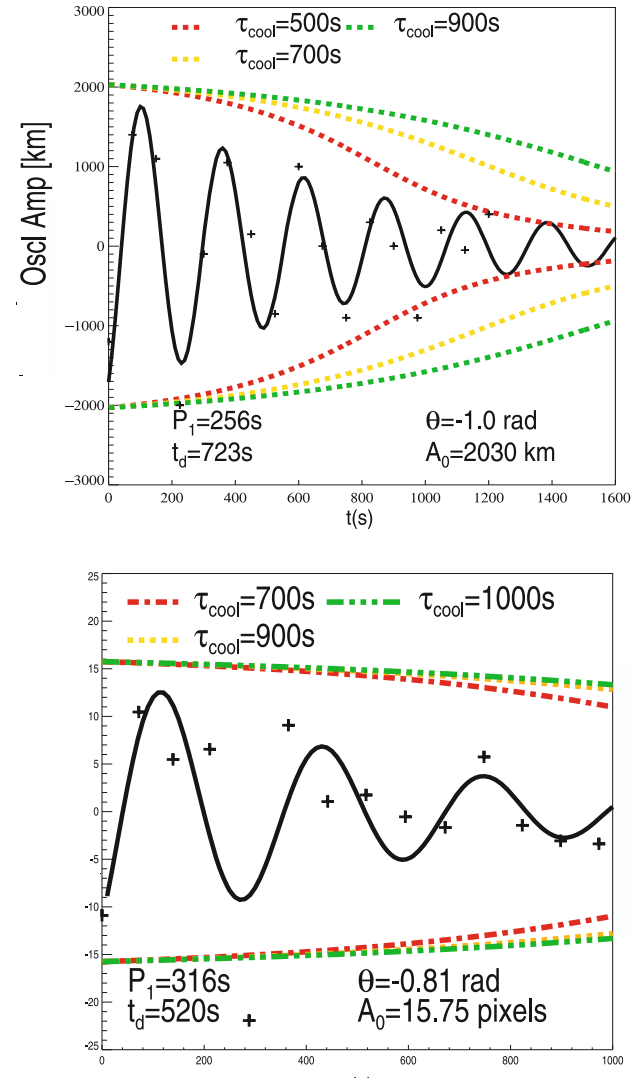

$t(s)$

Fig. 1. Examples of typical kink oscillations damped by radiation. The crosses are the observed data points (Nakariakov et al. 1999) and Aschwanden et al. (2002), solid black line is the fitted sine function Eq. (4). The various dashed lines are the envelope damping profiles due to cooling. In the text the individual plots are referred to as such, from left to right, top to bottom, $a, b, \ldots f$, respectively.

Table 1. Information about oscillating loops shown in Fig. 1.

\begin{tabular}{lcccc}
\hline \hline Panel & Date & Duration & $h_{\mathrm{a}}$ & Best fit $\tau_{\text {cool }}$ \\
\hline Fig. 1a & 14 July 1998 & $1500 \mathrm{~s}$ & $41 \mathrm{Mm}$ & $500 \mathrm{~s}$ \\
Fig. 1b & 14 July 1998(1f) & $1563 \mathrm{~s}$ & $49 \mathrm{Mm}$ & $500 \mathrm{~s}$ \\
Fig. 1c & 23 Nov 1998 & $3179 \mathrm{~s}$ & $134 \mathrm{Mm}$ & $1200 \mathrm{~s}$ \\
Fig. 1d & 14 July 1998 (1d) & $898 \mathrm{~s}$ & $46 \mathrm{Mm}$ & $700 \mathrm{~s}$ \\
Fig. 1e & 14 July 1998 (1g) & $1591 \mathrm{~s}$ & $38 \mathrm{Mm}$ & $500 \mathrm{~s}$ \\
Fig. 1f & 21 March 2001 & $2456 \mathrm{~s}$ & $135 \mathrm{Mm}$ & $1000 \mathrm{~s}$ \\
\hline
\end{tabular}

initial amplitude determined from the fitted analytical function, Eq. (4), for each oscillation and overplotted.

Most typical quoted examples in Fig. 1 show that the damping due to cooling (i.e. temporally varying background) is more than able to account for a significant amount of the observed decrease in amplitude. Analysing Fig. 1 suggests that the variable background (i.e. cooling of the plasma) offers a natural explanation for the damping of kink oscillations. In general, the degree of the match between theoretically predicted damping of the amplitudes of the oscillations and those detected by TRACE varies, depending upon the loop parameters. However, we find that in all cases, that we investigated, the dynamic background has at least some level of relevance for the damping. In Fig. 1a it can be seen that the theoretically predicted dynamic envelope profile of the damping due to cooling coincides very closely with that of the heuristically static fitted damping and is the best fit of all presented cases. The envelope from the damping due to cooling in Fig. 1b does not fit very closely the original damped sinusoidal profile by Aschwanden et al. (2002) but does include a number of the data points that are missed by this original profile. We then offer the view that the damping profile due to cooling can provide a better fit to the observed data points than the original heuristic fitted damping profile.

In Figs. 1c-e the best fit damping profile from the cooling appears to be able to match the heuristically static fitted damping profile less closely. However, in Fig. 1c the cooling profile provides a close fit for the oscillation from $1100 \mathrm{~s}$. Anyway, in all of these figures the best fit damping profile from the cooling does however encompasses some of the data points that are outside the envelope of the analytically fitted static damping profile. This could be evidence that the originally fitted damping profile is not the correct profile. Note that without error bars for the data, we cannot rule out these outlying points could fall within the fitted damping envelope. Unfortunately the error bars are not available in their observational reports. A different explanation for the difference between the best fit damping profile due to cooling and the analytically fitted damping profile in Figs. 1c-e can also be offered. For each of these oscillations, note that the timescale of the period, $P$, is close to that of the fitted damping time, $\tau_{\mathrm{d}}$, i.e. the damping is rapid compared to the period of the oscillation. The reason that we have a disagreement between these cooling and best fit damping profiles could be that the characteristic cooling is less than $500 \mathrm{~s}$. The practical restriction placed upon the applicable allowed values of the ratio $P / \tau_{\text {cool }}$ by the WKB method, means that damping profiles calculated for smaller values of $\tau_{\text {cool }}$ could have a pronounced approximation error and we do not calculate the profiles for less than $500 \mathrm{~s}$ here with the tools of the analytical theory. For such cases a full numerical simulation would be required. An alternative 

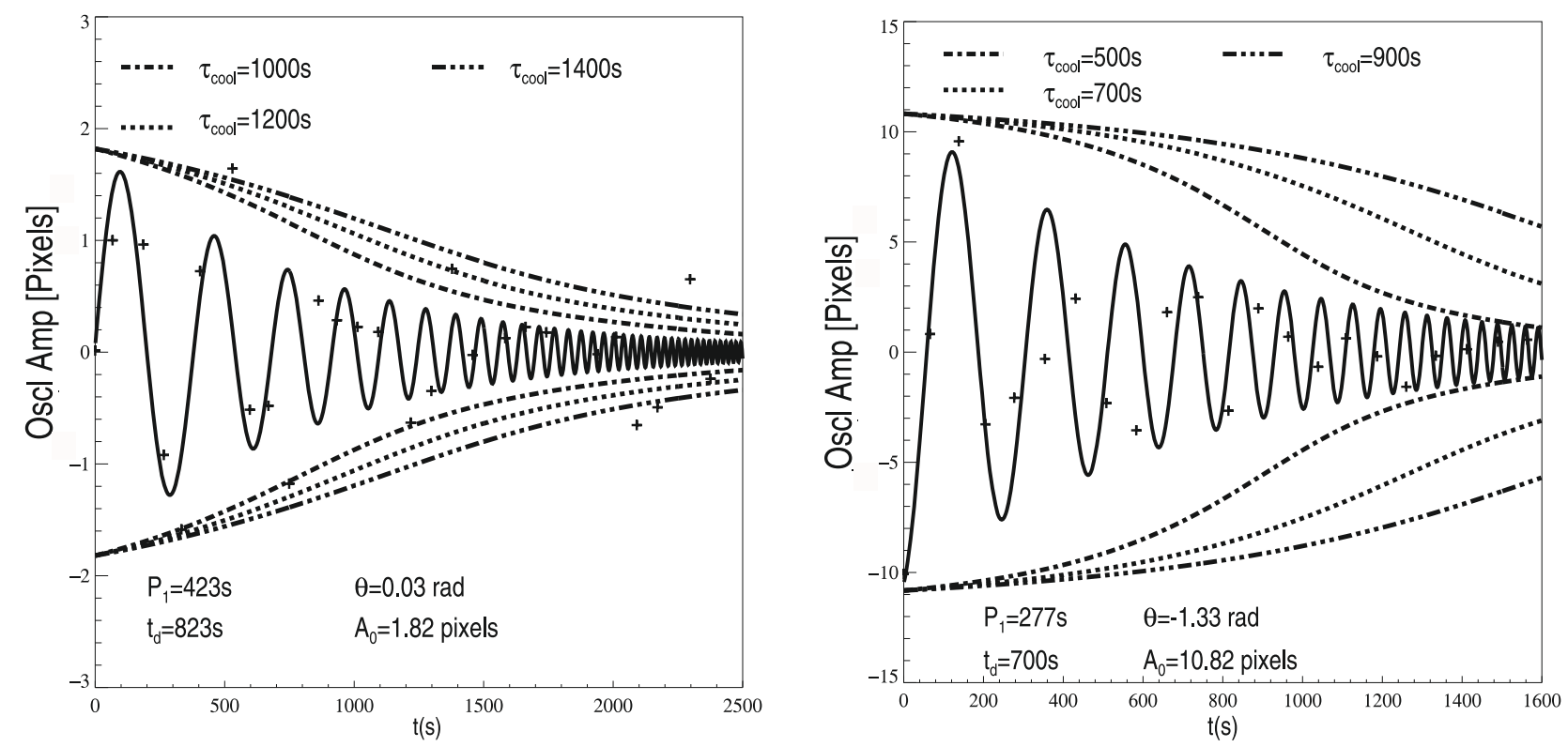

Fig. 2. In the left panel the oscillation in Fig. 1b is shown again here. Right panel shows Fig. 1e. Now plotted is the expected oscillation profile for the time dependent period substituted into the fitted sine function, Eq. (4).

explanation for the discrepancy between the calculated damping profiles and the observed profiles could be that another dissipative process other than the cooling, e.g. resonant absorption, has some important contribution to the damping of those particular observations. Even so, it is clear that the cooling could still provide a significant and dominant contribution to the damping for these examples. In Fig. 1f it is suggestive that the best fit damping profile from the cooling also does not fit well the observed damping of the oscillation. The heuristically static fitted damping profile, however, also does not fit a great number of the data points. This leads to the suggestion that the oscillation may need to be re-analysed or some existing physics is missing from the modelling.

\section{Issue on time-(in)dependent period}

In the previous section it was pointed out while the loop cools the period of the loop is also expected to change (see, e.g. Eq. (3)). In the reported observations of the damped kink oscillations analysed earlier by Nakariakov et al. (1999) and Aschwanden et al. (2002), the fitted sine function has a constant period (time independent) and apparently agrees with various degrees of success with the observations. However, it should be noted the cadence for the TRACE EUV imager is around $75 \mathrm{~s}$, which will place a lower limit on the periodicity that analysis will be able to determine from the observations. Let us now re-analyse these loop oscillations assuming that there is a variable background with allowed characteristic time scales relevant to loop oscillations. In the left panel of Fig. 2, applying the theory outlined in ME09, we now plot the expected change in period if the loop is cooling with a typical rate, say, $\tau_{\text {cool }}=1000 \mathrm{~s}$ and starts oscillating with the observed initial period $423 \mathrm{~s}$. The estimated damping time for this profile is the same as that for the original static fitted profile. It can be seen that the revised time-dependent quasi-sinusoidal profile fits the observations equally as well if not better than the previous fit with constant period. There is, however, a clearly identifiable $\sim 70 \%$ change predicted in the period, where the period at the end of the oscillation is around 100-120 s. A much improved cadence data is now required to make further progress and confirm the period variations.

In the right panel of Fig. 2 we provide another typical example. This time, however, we show the predicted period change for a cooling timescale of $\tau_{\text {cool }}=700 \mathrm{~s}$ and we estimate the fitted damping time so that it is also $700 \mathrm{~s}$. Again, this allows for a better fit between the observed data points and the analytically fitted profile of the oscillation. Comparing to Fig. 1e, it appears that the new fitted quasi-sinusoidal profile of the analytically evolving loop oscillation, with changing period and damping time of $700 \mathrm{~s}$ fits a greater number of the observed data points than the one of a static kink oscillating coronal loop. In both these examples the amplitude envelopes do not provide an exact fit to the observed damping profile but the changing period does provide a better fit for the data points. We suggest that it may then be better to calculate the contribution of cooling to the observed damping by first calculating the cooling time that corresponds to the best fit period change. Fitting the corresponding amplitude envelope for cooling will then show the contribution needed by other mechanisms to account for the observed damping fully.

In order to further support our dynamic modelling, it should be noted that a $35 \%$ decrease in period has already been tentatively reported in De Moortel et al. (2004) who re-analysed the results from Nakariakov et al. (1999). No explanation was provided for the decreasing period in the study of De Moortel et al. (2004). The time dependent background proposed here may offer the missing physics.

\section{Conclusions}

We conclude that the damping due to a dynamic background could have a dominant or at least significant influence on transverse oscillations in EUV coronal loops. So far this theory seems to describe best the observed features of damped kink oscillations and concludes an important aspect of solar magnetoseismology. The observed decay of these oscillations can be accurately predicted only if the reported dynamic nature of the background plasma is also adequately taken into account. The damping due to cooling (i.e. variable background) seems to be 


\section{R. J. Morton and R. Erdélyi: Cooling coronal loops}

a very plausible and natural physical mechanism in most of the reported cases of kink oscillations in loops. The variable background (i.e. time dependent) certainly captures an important feature seen in many of the oscillating post-flare coronal loops. This dissipative (and non-conservative) physical process manages to explain the damping of the loops without the need for an ansatz mechanism. Cooling occurs ubiquitously in the corona and is expected to occur after a flaring event in or close to the loop. The flare is a strong contender as the driver for the transversal oscillation, so there will be a high chance the loop is cooling while oscillating. In a number of the currently known observed cases investigated, here we found that the cooling can account almost entirely for the observed damping. However, it appears another mechanism(s) of damping may still be required so that the observed damping can be explained and accurately modelled in some cases. We suggest to generalise modelling efforts where both resonant absorption (i.e. the important radial stratification) and a time dependent variable background are both taken into account for oscillating coronal loops. We also suggest that a further observational signature of damping due to cooling would be the decreasing period of the oscillation. Initial evidence is provided in order to stimulate further investigation. Allowing the period of the oscillation to change appears to be able to match the observations better than static loop modelling (although empirical error estimates of the measured amplitudes are 20-40\% - private communication M. Aschwanden). Whether we will be able to detect the change in period with current cadences onboard satellites will remain to be seen.

Acknowledgements. R.E. acknowledges M. Kéray for patient encouragement. The authors are also grateful to NSF, Hungary (OTKA, Ref. No. K67746) and the Science and Technology Facilities Council (STFC), UK for the financial support they received.

\section{References}

Andries, J., van Doorsselaere, T., Roberts, B., et al. 2009, Space Sci. Rev., 149, 3 Arregui, I., Andries, J., Van Doorsselaere, T., Goossens, M., \& Poedts, S. 2007, A\&A, 463, 333

Aschwanden, M. J., \& Terradas, J. 2008, ApJ, 686, L127

Aschwanden, M. J., Fletcher, L., Schrijver, C. J., \& Alexander, D. 1999, ApJ, 520,880

Aschwanden, M. J., Nightingale, R. W., \& Alexander, D. 2000a, ApJ, 541, 1059

Aschwanden, M. J., Tarbell, T. D., Nightingale, R. W., et al. 2000b, ApJ, 535, 1047

Aschwanden, M. J., de Pontieu, B., Schrijver, C. J., \& Title, A. M. 2002, Sol. Phys., 206, 99

Bender, C. M., \& Orszag, S. A. 1978, Advanced Mathematical Methods for Scientists and Engineers (New York: McGraw-Hill)

De Moortel, I., Munday, S. A., \& Hood, A. W. 2004, Sol. Phys., 222, 203

Dymova, M. V., \& Ruderman, M. S. 2005, Sol. Phys., 229, 79

Erdélyi, R., \& Taroyan, Y. 2008, A\&A, 489, L49

Goossens, M., Andries, J., \& Aschwanden, M. J. 2002, A\&A, 394, L39

Goossens, M., Arregui, I., Ballester, J. L., \& Wang, T. J. 2008, A\&A, 484, 851

Jakimiec, J., Sylwester, B., Sylwester, J., et al. 1992, A\&A, 253, 269

Klimchuk, J. A., Karpen, J. T., \& Antiochos, S. K. 2010, ApJ, 714, 1239

López Fuentes, M. C., Klimchuk, J. A., \& Mandrini, C. H. 2007, ApJ, 657, 1127

McEwan, M. P., Donnelly, G. R., Díaz, A. J., \& Roberts, B. 2006, A\&A, 460, 893

Morton, R. J., \& Erdélyi, R. 2009, ApJ, 707, 750

Nagata, S., Hara, H., Kano, R., et al. 2003, ApJ, 590, 1095

Nakariakov, V. M., Ofman, L., Deluca, E. E., Roberts, B., \& Davila, J. M. 1999, Science, 285,862

Ofman, L., \& Wang, T. J. 2008, A\&A, 482, L9

Reale, F., Peres, G., Serio, S., DeLuca, E. E., \& Golub, L. 2000, ApJ, 535, 412

Ruderman, M. S., \& Roberts, B. 2002, ApJ, 577, 475

Ruderman, M. S., \& Erdélyi, R. 2009, Space Sci. Rev., 149, 199

Taroyan, Y., Erdélyi, R., Wang, T. J., \& Bradshaw, S. J. 2007, ApJ, 659, L173

Ugarte-Urra, I., Warren, H. P., \& Brooks, D. H. 2009, ApJ, 695, 642

Verth, G., \& Erdélyi, R. 2008, A\&A, 486, 1015

Winebarger, A. R., \& Warren, H. P. 2005, ApJ, 626, 543 